\title{
1 Barley Long Non-Coding RNAs and Their Tissue-Specific Co-expression Pattern \\ 2 with Coding-Transcripts
}

3

4 Gökhan Karakülah ${ }^{1}$ and Turgay Unver ${ }^{1,2} *$

5

$6 \quad{ }^{1}$ İzmir International Biomedicine and Genome Institute (iBG-izmir), Dokuz Eylül

7 University, 35340, İnciralt1, İzmir, Turkey

$8{ }^{2}$ Present address: Egitim Mah., Ekrem Guer Sok. 26/3, 35340, Balcova, İzmir, Turkey

9

$10 *$ To whom correspondence should be addressed. Tel: +90 232412 6535; Fax: +90 232

11412 6509; Email: turgayunver@icloud.com

12

13 


\section{Abstract}

2 Long non-coding RNAs (lncRNA) with non-protein or small peptide-coding potential

3 transcripts are emerging regulatory molecules. With the advent of next-generation

4 sequencing technologies and novel bioinformatics tools, a tremendous number of

5 lncRNAs has been identified in several plant species. Recent reports demonstrated roles

6 of plant lncRNAs such as development and environmental response. Here, we reported

7 a genome-wide discovery of $\sim 8,000$ barley lncRNAs and measured their expression

8 pattern upon excessive boron (B) treatment. According to the tissue-based comparison,

9 leaves have a greater number of B-responsive differentially expressed lncRNAs than the

10 root. Functional annotation of the coding transcripts, which were co-expressed with

11 IncRNAs, revealed that molecular function of the ion transport, establishment of

12 localization, and response to stimulus significantly enriched only in the leaf. On the

13 other hand, 32 barley endogenous target mimics (eTM) as IncRNAs, which potentially

14 decoy the transcriptional suppression activity of 18 miRNAs, were obtained. Presented

15 data including identification, expression measurement, and functional characterization

16 of barley lncRNAs suggest that B-stress response might also be regulated by lncRNA

17 expression via cooperative interaction of miRNA-eTM-coding target transcript

18 modules.

20 Keywords: Boron, endogenous target mimicry, Hordeum vulgare, long non-coding

21 RNA, novel transcript discovery and annotation 


\section{1. Introduction}

3 Long non-coding RNAs (lncRNA) are known as non-protein coding transcripts longer

4 than $200 \mathrm{nt}$. Although, the information about their functions is so limited, studies

5 revealed that they have several direct and indirect roles in the transcriptional, post-

6 transcriptional or post-translational processes such as gene expression, chromatin

7 modification, transcriptional regulation, and conformational changes in proteins

8 (reviewed by Liu et al., 2015). They act as the precursor of micro-RNA (miRNA) and

9 short interfering-RNA (siRNA). For instance, five lncRNA in Arabidopsis (npc34,

10 npc351, npc375, npc520, and npc523) matched with 24-nt siRNAs from both strands,

11 suggesting these lncRNAs are siRNA precursor. Moreover, it was reported that plant

12 miRNAs, miR869a and miR160c, which were derived from lncRNAs of npc83 and

13 npc521, respectively (Ben Amor et al., 2009). Additionally, it was also discovered that

14 miR675 is derived from a mouse lncRNA, H19, and extensively expressed in

15 embryonic liver (Dey et al., 2014; Keniry et al., 2012).

17 Recently, short peptide-coding sequences were discovered in the non-coding regions of

18 plant primary-miRNAs (pri-miRNA) and called as miRNA-encoded peptide (miPEP),

19 which increases the transcription of pri-miRNA (Lauressergues et al., 2015; Waterhouse

20 and Hellens, 2015). Therefore, it was suggested that some plant lncRNAs might have

21 peptide coding potential (Liu et al. 2015). In addition, miRNA activity can be regulated

22 by endogeneous target mimicry (eTM) molecules, being a type of lncRNAs (Karakulah

23 et al., 2016). Such an example, an endogenous lncRNA called Induced by Phosphate

24 Starvation 1 (IPS1) of Arabidopsis thaliana binds to miR399 to inhibit the cleavage of 
1 the miR399 target transcript (Franco-Zorrilla et al., 2007). Other than these functions,

2 plant lncRNAs were found to be involved in many regulatory mechanisms, such as

3 histone modeling (Heo and Sung, 2011), promoter modification (Ding et al., 2012;

4 Zhou et al., 2012), protein re-localization (Sousa et al., 2001), and alternative splicing

5 (Bardou et al., 2014).

7 To date, lncRNAs have been identified extensively in mammals, in which human

8 genome includes more than 56,000, and mice have almost 46,000 lncRNAs (Xie et al.,

9 2014). Publicly available databases such as LncRNAdb (http://lncrnadb.com), a

10 database for functional lncRNAs, harbor functionally annotated lncRNAs, of the

11 majority belong to the human. A few databases were so far released for plant lncRNAs;

12 such as the Green Non-Coding Database (GreeNC, http://greenc.sciencedesigners.com)

13 (Paytuvi Gallart et al., 2016) and CANTATAdb (http://cantata.amu.edu.pl) (Szczesniak

14 et al., 2016), which provides information for around 45,117 lncRNAs from several plant

15 species. RNA-sequencing or deep transcriptome analysis is an important technology,

16 which provides information not only for protein coding transcripts, but also for non-

17 coding RNAs (such as miRNA, siRNA, piwi-interacting RNA (piRNA), and small-

18 nucleolar RNA (snoRNA) as well as lncRNAs). In addition, it allows distinguishing

19 lncRNAs expressed in different tissues or cells.

21 Boron (B) is an essential micronutrient for plants, and its unfavorable concentration

22 negatively affects plant growth and productivity where the soils having with insufficient

23 or excess B (Unver et al., 2008). The range of sufficient B concentration in soil is so

24 limited, thus generally plants suffer from either B-deficiency or -toxicity problem that is 
1 common in agricultural soils of around the world (Camacho-Cristobal et al., 2015).

2 However, plants growing in B-contaminated soils must tolerate the excess level of B to

3 survive. In the last decade, many studies conducted to understand the cellular

4 mechanisms underlying to balance cellular B content in plants (Miwa and Fujiwara,

5 2010; Tombuloglu et al., 2015). Facilitated transport of boric acid (a regular form of B

6 in soil) by transporter channels was suggested to be the molecular regulatory

7 mechanism. Several B-importer and exporter proteins have recently been identified as

8 B-transporters to regulate its cellular homeostasis (Miwa and Fujiwara, 2010). On the

9 other hand, novel sequencing-based approaches to discover the transcriptional response

10 at genome-wide level are being extensively utilized in plants faced with unfavorable

11 environmental conditions. In this context, to quantify gene expression and to annotate

12 coding-transcripts, we performed a high-throughput genome-wide transcriptome

13 analysis on barley tissues treated with excess B (1 mM), previously (Tombuloglu et al.,

14 2015). In addition, we also screened B-responsive miRNA expression pattern (Ozhuner

15 et al., 2013); identified MYB type transcription factors (TF) (Tombuloglu et al., 2013)

16 and water channel Aquaporins (AQP) (Tombuloglu et al., 2016) to understand B

17 homeostasis of barley. These studies helped to observe the main or possible players

18 involved in B regulation. Besides the emerging evidences suggested that the molecular

19 regulatory mechanism is so complicated and not only limited to activity of those coding

20 transcripts. LncRNAs being the new players were also be discovered as regulatory

21 molecules on the regulation of gene expressions.

23 To date, a large set of RNA-seq libraries was used to identify lncRNAs in genome-wide

24 scale or tissue/condition/inoculation-specific manner (Chen et al., 2016; Li et al., 2014; 
1 Liu et al., 2012). Stress-responsive lncRNAs were examined from the RNA-seq data of

2 the plants under the distinct type of stress conditions as well (reviewed by Chekanova,

3 2015; Shafiq et al., 2016; Zhang et al., 2013). Qi et al., 2013 identified 584 lncRNAs,

4 which were responsive to drought stress in foxtail millet. 125 putative lncRNAs were

5 identified in wheat, responsive to powdery mildew infection and heat stress (Xin et al.,

6 2011). Detailed examination of a large set of poplar (Populus trichocarpa) RNA-seq

7 data revealed 504 lncRNAs in response to drought (Shuai et al., 2014). Additionally,

8 Huang et al reported over 12,000 barley lncRNAs, of them 604 were Fusarium head

9 blight inoculation responsive (Huang et al., 2016). However, no such a study to profile

10 expression level of lncRNAs under the B-excess as one of the abiotic stress conditions

11 was conducted till now. In this study, we identified and quantitatively compared the

12 expression of B-responsive barley lncRNAs from four transcriptome datasets. Tissue-

13 specific (root and leaf) and excess B-responsive lncRNAs, which were co-expressed

14 with coding-transcripts, discovered and comprehensively analyzed. 


\section{2. Materials and Methods}

3 2.1. Identification of barley lncRNAs

4 To study global expression profiling of barley lncRNAs under boron stress condition,

5 we utilized four transcriptome libraries each including pooled RNAs from tree

6 biological replicate previously generated by our group (SUB337351 and SUB2170217)

7 (Tombuloglu et al., 2015). Briefly, a total of 208,249,690 clean sequencing reads from

8 four paired-end libraries (50_leaf; 52, 422,032, 50_root; 52,168,358, 1000_leaf;

$952,305,062$, and 1000_root, 51,354,238) were utilized in this study. We first removed

10 the adapter sequences and low-quality reads from the sequencing reads with

11 Trimmomatic v0.36 (Bolger et al., 2014), and these clean reads were aligned to the

12 barley reference genome (ASM32608v1 assembly) by TopHat2 v2.1.1 with default

13 parameters (Kim et al., 2013). Afterward, genome-aligned reads were assembled $a b$

14 initio using popular transcriptome analysis suit Cufflinks v2.2.1 (Trapnell et al., 2010)

15 to build potential transcript structures. All gene transfer format (GTF) files produced in

16 the assembly step were merged with Cuffmerge tool, and transcript features were

17 queried against the Ensembl Plant database (release 33) (Kersey et al., 2016) by

18 Cuffcompare to discover previously unannotated transcript sequences. Among all un-

19 annotated transcripts, including coding and non-coding sequences, we obtained

20 lncRNAs as follows: (i) we first tested coding potential of each transcript individually

21 using TransDecoder (https://transdecoder.github.io/), and filtered out those with an open

22 reading frame having more than 100 amino acids, (ii) we then removed transcripts

23 which were shorter than 200 nucleotides in length, (iii) to remove housekeeping

24 lncRNA species, we queried all potential lncRNAs against to non-coding RNA family

25 database, Rfam (v12.1) (Nawrocki et al., 2015), with Infernal tool (v1.1.1; cutoff E-

26 value $\leq$ 0.001) (Nawrocki et al., 2009). Then, potential miRNA precursors, tRNAs etc

27 were removed and were not included for further analysis, and (iv) we aligned all

28 potential IncRNA transcripts to the Swiss-Prot database (release 2017_01) (The

29 UniProt, 2017) using Blastx (v2.5.0; cutoff E-value $\leq$ 0.001) (Camacho et al., 2009) to

30 eliminate transcripts with potential protein-coding ability. 


\section{2.2. Expression pattern analysis of coding and non-coding RNAs}

2 Transcript abundances in each library were measured with Kallisto v0.43.0 (Bray et al.,

3 2016). Then the transcripts expressed <1 $\underline{\text { Transcripts }} \underline{\text { Per }} \underline{\text { Million }}$ (TPM) in all libraries

4 were considered as transcriptional noise and were removed from further downstream

5 analysis steps. Differentially expressed transcripts within each group (leaf and root

6 samples) were determined by calculating fold changes of TPM values in RNA-seq

7 datasets. Transcripts with differential expression values $\geq 2$ fold-changes in compared

8 datasets were classified as boron responsive. The Gene Ontology (GO) analysis of

9 differentially expressed genes was performed using online GO analysis toolkit, agriGO

10 applying default parameters (Du et al., 2010).

12 2.3. Co-expression analysis of IncRNAs with coding mRNAs and prediction of

13 endogenous target mimicry (eTM) sequences

14 We predicted putative functions of differentially expressed lncRNAs with "guilt-by15 association" approach, which employed in previous studies for lncRNA annotation

16 (Rinn and Chang, 2012) (Guo et al., 2013) (D'Haene et al., 2016). To reveal potential

17 lncRNA-mRNA associations, we identified co-expressed mRNA-lncRNA pairs with

18 Spearman's correlation test in R v3.1.0 statistical computation environment (Team,

19 2016). Then, co-localized mRNA-lncRNA pairs on the reference genome were

20 identified with Bedtools v2.25.0 (Quinlan, 2014). We considered the mRNA-lncRNA

21 pair as co-expressed if the Spearmen's rho is equal or greater than $0.90(\mathrm{p}$-val<0.01)

22 between the expression values of coding and lncRNA transcripts, and as co-localized

23 when the distance between two transcripts were less than $10 \mathrm{~kb}$..To dissect putative

24 eTM sequences among the transcripts annotated as lncRNA, we employed our analysis 
1 pipeline previously introduced by our group (Karakulah et al., 2016). In the eTM

2 sequences analysis pipeline, we utilized mature miRNA sequences of barley collected

3 from miRBase (release 21) (Griffiths-Jones, 2006). To identify potential target

4 transcripts of barley miRNAs, we utilized psRNATarget, an online miRNA target

5 analysis tool (Dai and Zhao, 2011) as previously described previously (Akdogan et al.,

6 2016; Bakir et al., 2016; Eldem et al., 2012; Inal et al., 2014; Yanik et al., 2013). 


\section{3. Results and discussion}

2

\section{3.1. Barley lncRNA identification}

4 After the adapter sequence trimming and removal of low-quality reads, the mean library

5 size of four sequencing libraries included in the study was over 20 million (min:

6 18223418, max: 22171430). Additionally, we observed an average of $82.35 \%$ (min:

$7 \quad 78.7 \%$, max: $90.9 \%$ ) overall read mapping for the alignment step, and considered all

8 sequencing libraries had sufficient quality to perform an $a b$ initio transcriptome

9 reconstruction analysis. The run of Cufflinks pipeline was led to the identification of as

10 many as 34,000 previously unannotated intergenic transcripts, of which 10,439 were the

11 lack of coding potential and more than 200 bp in length (Table S1). When we filtered

12 out the transcripts expressed at low levels ( $<1$ TPM in all samples), we obtained 8,009

13 intergenic putative lncRNAs in the final list, which were distributed almost equally to

14 all barley chromosomes (Figure 1A). However, chromosome 2 was observed to be the

15 richest one in terms of the total number of lncRNAs it harbors. In this study, a total

16 number of $\sim 8,000$ barley lncRNAs were identified which is smaller than that of human

$17(\sim 56,000)$ (Xie et al., 2014$)$ and mouse $(\sim 46,000)$; higher than fruit fly $(\sim 3,300)$ (Chen

18 et al., 2016), and poplar (2,542) (Shuai et al., 2014) (Table S1). Actual numbers of

19 lncRNAs can be altered depending on sample examined. In this analysis, four RNA-Seq

20 libraries were used to detect total lncRNAs. More lncRNAs can be found from barley

21 genome by increasing the number of RNA-Seq sets from distinct tissues and/or

22 conditions. In general, low expression levels of most lncRNAs compared to protein-

23 coding genes make it more difficult to detect lncRNAs (Mercer et al., 2011). Generally,

24 they are excluded from the total lncRNA pool resulting fluctuations of total lncRNA 
1 number. But it is important to note that lncRNAs with low expression may have a big

2 impact, thus extensive and a deep pipeline is required to extract lncRNA, which may

3 possess important biological functions. In general, the distribution of lncRNAs to the

4 barley chromosomes is proportional with its chromosome sizes, except chromosome 2,

5 which includes the highest number (Fig1A).

7 3.2. Expression pattern of barley lncRNAs and coding transcripts upon excess B-

8 treatment

9 As the expression profiles of lncRNAs in root and leaf samples were examined, it was

10 determined that expression levels of lncRNAs in the samples collected from same tissue

11 were similar to one another (Figure 1B). The hierarchical clustering analysis, however,

12 revealed that particular lncRNA clusters were expressed at relatively higher levels

13 specific to tissue types (Figure 1C). Differential expression analysis of both coding and

14 lncRNA transcripts showed that there was 2 fold or more change (up- or down-

15 regulation) in the expression of the vast amount of transcripts in response to boron

16 stress in leaves and root tissues (Table S2). We observed that the number of common

17 coding transcripts that were differentially regulated in both tissues was 517; in addition,

18 the total number of differentially expressed coding transcripts in the leaf tissue was

19 roughly doubled as compared to root tissue (Figure 2A). Similar to the differential

20 expression analysis of coding transcripts, we detected a greater number of up- or

21 downregulated lncRNAs in the leaves than the root samples (Figure 2B).

23 3.3. Functional annotation of IncRNAs co-expressed with coding transcripts 
1 The GO enrichment analysis of differentially expressed coding transcripts (Table S3)

2 revealed that ion transport (GO:0006811), establishment of localization (GO:0051234),

3 and response to stimulus (GO:0050896) terms significantly enriched (FDR<0.05) only

4 in the leaf samples (Figure 2C). In addition to this, molecular function terms of ligase

5 activity (GO:0016874) and cysteine-type peptidase activity (GO:0008234) were

6 significant and specific to the leaves (Figure 2D). Based on the co-expression and co-

7 localization analysis of barley lncRNAs and coding transcripts, we observed potential

8 lncRNAs in association with the ion transports, establishment of localization, and

9 response to stimulus related transcripts (Figures 3 and 4). We determined 6 lncRNAs,

10 which strongly linked to ion transport related genes (Figure 3A). However, only one

11 lncRNA (TCONS_00061958) was showing $\geq 2$ fold expressional change in both the

12 leaf and root samples (Figure 3B). A great majority of response to stimulus related

$13 \operatorname{lncRNAs}$ increased ( $\geq 2$ fold) their expression in leaf samples upon the boron exposure

14 (Figure 3C-D).

15 Additionally, we detected 12 lncRNA sequences, which had similar expression patterns

16 and co-localized with "establishment of localization" related genes (Figure 4). We also

17 detected that some of the lncRNAs were only differentially expressed in tissue-specific

18 manner such as TCONS_00002116 expression in leaf under B-excess (Figure 4B).

19 However, TCONS_00061958 was found to be differentially regulated in both leaf and

20 root tissues. Studies also revealed the differential expression pattern of plant lncRNAs

21 such as Arabidopsis, wheat, and poplar in response to biotic and abiotic stresses (Liu et

22 al., 2012; Shuai et al., 2014; Xin et al., 2011). Here, we identified that barley boron-

23 responsive lncRNAs are expressed in the tissue-specific manner (Figures 2-4 and Table

24 S2). According to GO term enrichment analysis, differentially expressed coding 
1 transcripts were categorized into three biological processes: response to stimulus (50

2 transcripts), ion transport (17 transcripts), and establishment of localization (52

3 transcripts), which were leaf specific in this analysis (Fig 2C and Table S3). These

4 findings were consistent with our previous report (Tombuloglu et al., 2015), where the

5 biological process, ion transport, and establishment of localization categories were

6 found to be enriched in both leaf and root tissues.

7

$8 \quad 3.4$. eTM sequence discovery

9 Here, we predicted 32 barley eTMs, which might decoy the transcriptional suppression

10 activity of 18 miRNAs, including conserved barley miRNAs such as miR159a,

11 miR166a, and miR399 (Table 1 and Table S4). In the GO enrichment analysis of

12 miRNA target genes using the agriGO tool, we found 102 significant $($ FDR $<0.05)$

13 different GO terms in three domains, consisting of molecular function, biological

14 process, and cellular component (Table S5). Among the terms, the most significant one

15 was "protein amino acid phosphorylation" term (GO:0006468, FDR=4.70E-58). Our

16 findings in eTM analysis suggest that barley lncRNAs might regulate several distinct

17 cellular and molecular processes via mimicking specific miRNA target transcripts. As it

18 was previously reported by recent studies, lncRNAs might behave as a mimicry-

19 transcript that targeted by miRNAs and fate it to degradation (Juan et al., 2013). It was

20 firstly reported in Arabidopsis, over-expression of non-coding gene IPS1 suppressed the

21 miR399 expression that resulted in elevated expression of the miR399 target (Franco-

22 Zorrilla et al., 2007). On the other hand, we determined and measured the boron-

23 responsive barley miRNAs (Ozhuner et al., 2013). Accordingly, miR5049 was down-

24 regulated in B-stressed leaf (three times than control leaf). Also, miR399 was over- 
1 expressed in leaf and suppressed in root tissue upon B-exposure (three times of each

2 tissue than that of control). In this study, miR5049 and miR399 were also found to be as

3 regulated miRNAs upon B-treatment by eTM analysis (Table S4) where

4 TCONS_00032652 and TCONS_00043651 have predicted as the target mimic

5 sequences, which are able to decoy the miRNA activities, respectively. Thus, expression

6 of transcripts targeted by these miRNAs might be altered due to differential expressions

7 of lncRNAs. Phosphate transporter 2 (PHO2) and putative ubiquitin-conjugating

8 enzyme $(U B C)$ were found to be the target genes of miR399. Also, tubby protein-like

9 transcript was determined as the miR5049 target. In the transcriptome analysis,

10 ubiquitin carboxyl-terminal hydrolase gene was highly up-regulated in leaf tissue upon

11 excess B treatment (Tombuloglu et al., 2015). Interestingly, expression profiles of

12 miRNA and its corresponding lncRNA target mimic transcript provide insights into the

13 regulation of B-stress in plants. For instance, lncRNA TCONS_00043651, a potential

14 target mimic sequence of miR399, up-regulated in roots (three times than that of

15 control) upon B-exposure. Oppositely, miR399 expression was reflected with the same

16 pattern in a negative direction (three times down-regulated). Similarly, five times

17 increase of lncRNA TCONS_00043651 in leaf tissues may prevent the expression of

18 miR399, which was up-regulated only three times (Table S2). These preliminary results

19 suggest that boron regulation can be cooperatively controlled by the interaction of

20 miRNA-eTM (lncRNA)- coding target transcript modules. 


\section{4. Conclusion}

2 With the development of next-generation sequencing technologies and advancement in

3 bioinformatics, more transcriptional datasets were generated including the units with

4 little or no protein-coding potential. In recent years, the lncRNAs considered as

5 regulatory molecules in several bioprocesses. Though a large number of lncRNA

6 transcripts were identified in plants, no such genome-wide study was conducted for

7 barley as an important crop. Another missing biological hypothesis is that the possible

8 involvement of lncRNAs in boron-response mechanism. Here, we reported the genome-

9 wide discovery of $\sim 8,000$ barley lncRNAs and measured their expression pattern upon

10 excessive boron (B) treatment. Furthermore, we functionally annotated the coding

11 transcripts, which are co-expressed with lncRNAs and showed that cooperative

12 interaction of miRNA-eTM (lncRNA)- coding target transcript modules might regulate

13 the boron-response in barley. 


\section{Acknowledgements}

2 The authors greatly appreciate ICGEB with grant no CRP/TUR16-03.

3

\section{Author Contributions}

5 GK and TU organized the study. GK performed analyses and TU interpreted the data.

$6 \quad$ GK and TU wrote the manuscript.

7

\section{Conflicts of Interest}

9 The authors declare no conflict of interest.

10

11 


\section{References}

Akdogan, G., Tufekci, E. D., Uranbey, S., Unver, T., 2016. miRNA-based drought regulation in wheat. Funct Integr Genomics 16, 221-33, doi:10.1007/s10142015-0452-1.

Bakir, Y., Eldem, V., Zararsiz, G., Unver, T., 2016. Global Transcriptome Analysis Reveals Differences in Gene Expression Patterns Between Nonhyperhydric and Hyperhydric Peach Leaves. Plant Genome 9, doi:10.3835/plantgenome2015.09.0080.

Bardou, F., Ariel, F., Simpson, C. G., Romero-Barrios, N., Laporte, P., Balzergue, S., Brown, J. W., Crespi, M., 2014. Long noncoding RNA modulates alternative splicing regulators in Arabidopsis. Dev Cell 30, 166-76, doi:10.1016/j.devcel.2014.06.017.

Ben Amor, B., Wirth, S., Merchan, F., Laporte, P., d'Aubenton-Carafa, Y., Hirsch, J., Maizel, A., Mallory, A., Lucas, A., Deragon, J. M., Vaucheret, H., Thermes, C., Crespi, M., 2009. Novel long non-protein coding RNAs involved in Arabidopsis differentiation and stress responses. Genome Res 19, 57-69, doi:10.1101/gr.080275.108.

Bolger, A. M., Lohse, M., Usadel, B., 2014. Trimmomatic: a flexible trimmer for Illumina sequence data. Bioinformatics 30, 2114-20, doi:10.1093/bioinformatics/btu170.

Bray, N. L., Pimentel, H., Melsted, P., Pachter, L., 2016. Near-optimal probabilistic RNA-seq quantification. Nat Biotechnol 34, 525-7, doi:10.1038/nbt.3519.

Camacho, C., Coulouris, G., Avagyan, V., Ma, N., Papadopoulos, J., Bealer, K., Madden, T. L., 2009. BLAST+: architecture and applications. BMC Bioinformatics 10, 421, doi:10.1186/1471-2105-10-421.

Camacho-Cristobal, J. J., Martin-Rejano, E. M., Herrera-Rodriguez, M. B., NavarroGochicoa, M. T., Rexach, J., Gonzalez-Fontes, A., 2015. Boron deficiency inhibits root cell elongation via an ethylene/auxin/ROS-dependent pathway in Arabidopsis seedlings. J Exp Bot 66, 3831-40, doi:10.1093/jxb/erv186.

Chekanova, J. A., 2015. Long non-coding RNAs and their functions in plants. Curr Opin Plant Biol 27, 207-16, doi:10.1016/j.pbi.2015.08.003.

Chen, M. J., Chen, L. K., Lai, Y. S., Lin, Y. Y., Wu, D. C., Tung, Y. A., Liu, K. Y., Shih, H. T., Chen, Y. J., Lin, Y. L., Ma, L. T., Huang, J. L., Wu, P. C., Hong, M. Y., Chu, F. H., Wu, J. T., Li, W. H., Chen, C. Y., 2016. Integrating RNA-seq and ChIP-seq data to characterize long non-coding RNAs in Drosophila melanogaster. BMC Genomics 17, 220, doi:10.1186/s12864-016-2457-0.

D'Haene, E., Jacobs, E. Z., Volders, P. J., De Meyer, T., Menten, B., Vergult, S., 2016. Identification of long non-coding RNAs involved in neuronal development and intellectual disability. Sci Rep 6, 28396, doi:10.1038/srep28396.

Dai, X., Zhao, P. X., 2011. psRNATarget: a plant small RNA target analysis server. Nucleic Acids Res 39, W155-9, doi:10.1093/nar/gkr319.

Dey, B. K., Pfeifer, K., Dutta, A., 2014. The H19 long noncoding RNA gives rise to microRNAs miR-675-3p and miR-675-5p to promote skeletal muscle differentiation and regeneration. Genes Dev 28, 491-501, doi:10.1101/gad.234419.113. 
Ding, J., Shen, J., Mao, H., Xie, W., Li, X., Zhang, Q., 2012. RNA-directed DNA methylation is involved in regulating photoperiod-sensitive male sterility in rice. Mol Plant 5, 1210-6, doi:10.1093/mp/sss095.

Du, Z., Zhou, X., Ling, Y., Zhang, Z. H., Su, Z., 2010. agriGO: a GO analysis toolkit for the agricultural community. Nucleic Acids Research 38, W64-W70, doi:10.1093/nar/gkq310.

Eldem, V., Celikkol Akcay, U., Ozhuner, E., Bakir, Y., Uranbey, S., Unver, T., 2012. Genome-wide identification of miRNAs responsive to drought in peach (Prunus persica) by high-throughput deep sequencing. PLoS One 7, e50298, doi:10.1371/journal.pone.0050298.

Franco-Zorrilla, J. M., Valli, A., Todesco, M., Mateos, I., Puga, M. I., Rubio-Somoza, I., Leyva, A., Weigel, D., Garcia, J. A., Paz-Ares, J., 2007. Target mimicry provides a new mechanism for regulation of microRNA activity. Nat Genet 39, 1033-7, doi:10.1038/ng2079.

Griffiths-Jones, S., 2006. miRBase: the microRNA sequence database. Methods Mol Biol 342, 129-38, doi:10.1385/1-59745-123-1:129.

Guo, X., Gao, L., Liao, Q., Xiao, H., Ma, X., Yang, X., Luo, H., Zhao, G., Bu, D., Jiao, F., Shao, Q., Chen, R., Zhao, Y., 2013. Long non-coding RNAs function annotation: a global prediction method based on bi-colored networks. Nucleic Acids Res 41, e35, doi:10.1093/nar/gks967.

Heo, J. B., Sung, S., 2011. Vernalization-mediated epigenetic silencing by a long intronic noncoding RNA. Science 331, 76-9, doi:10.1126/science.1197349.

Huang, Y., Li, L., Smith, K. P., Muehlbauer, G. J., 2016. Differential transcriptomic responses to Fusarium graminearum infection in two barley quantitative trait loci associated with Fusarium head blight resistance. BMC Genomics 17, 387, doi:10.1186/s12864-016-2716-0.

Inal, B., Turktas, M., Eren, H., Ilhan, E., Okay, S., Atak, M., Erayman, M., Unver, T., 2014. Genome-wide fungal stress responsive miRNA expression in wheat. Planta 240, 1287-98, doi:10.1007/s00425-014-2153-8.

Juan, L., Wang, G., Radovich, M., Schneider, B. P., Clare, S. E., Wang, Y., Liu, Y., 2013. Potential roles of microRNAs in regulating long intergenic noncoding RNAs. BMC Med Genomics 6 Suppl 1, S7, doi:10.1186/1755-8794-6-S1-S7.

Karakulah, G., Yucebilgili Kurtoglu, K., Unver, T., 2016. PeTMbase: A Database of Plant Endogenous Target Mimics (eTMs). PLoS One 11, e0167698, doi:10.1371/journal.pone.0167698.

Keniry, A., Oxley, D., Monnier, P., Kyba, M., Dandolo, L., Smits, G., Reik, W., 2012. The H19 lincRNA is a developmental reservoir of miR-675 that suppresses growth and Igf1r. Nat Cell Biol 14, 659-65, doi:10.1038/ncb2521.

Kersey, P. J., Allen, J. E., Armean, I., Boddu, S., Bolt, B. J., Carvalho-Silva, D., Christensen, M., Davis, P., Falin, L. J., Grabmueller, C., Humphrey, J., Kerhornou, A., Khobova, J., Aranganathan, N. K., Langridge, N., Lowy, E., McDowall, M. D., Maheswari, U., Nuhn, M., Ong, C. K., Overduin, B., Paulini, M., Pedro, H., Perry, E., Spudich, G., Tapanari, E., Walts, B., Williams, G., Tello-Ruiz, M., Stein, J., Wei, S., Ware, D., Bolser, D. M., Howe, K. L., Kulesha, E., Lawson, D., Maslen, G., Staines, D. M., 2016. Ensembl Genomes 2016: more genomes, more complexity. Nucleic Acids Res 44, D574-80, doi:10.1093/nar/gkv1209. 
Kim, D., Pertea, G., Trapnell, C., Pimentel, H., Kelley, R., Salzberg, S. L., 2013. TopHat2: accurate alignment of transcriptomes in the presence of insertions, deletions and gene fusions. Genome Biol 14, R36, doi:10.1186/gb-2013-14-4r36.

Lauressergues, D., Couzigou, J. M., Clemente, H. S., Martinez, Y., Dunand, C., Becard, G., Combier, J. P., 2015. Primary transcripts of microRNAs encode regulatory peptides. Nature 520, 90-3, doi:10.1038/nature14346.

Li, L., Eichten, S. R., Shimizu, R., Petsch, K., Yeh, C. T., Wu, W., Chettoor, A. M., Givan, S. A., Cole, R. A., Fowler, J. E., Evans, M. M., Scanlon, M. J., Yu, J., Schnable, P. S., Timmermans, M. C., Springer, N. M., Muehlbauer, G. J., 2014. Genome-wide discovery and characterization of maize long non-coding RNAs. Genome Biol 15, R40, doi:10.1186/gb-2014-15-2-r40.

Liu, J., Jung, C., Xu, J., Wang, H., Deng, S., Bernad, L., Arenas-Huertero, C., Chua, N. H., 2012. Genome-wide analysis uncovers regulation of long intergenic noncoding RNAs in Arabidopsis. Plant Cell 24, 4333-45, doi:10.1105/tpc.112.102855.

Liu, X., Hao, L., Li, D., Zhu, L., Hu, S., 2015. Long non-coding RNAs and their biological roles in plants. Genomics Proteomics Bioinformatics 13, 137-47, doi:10.1016/j.gpb.2015.02.003.

Mercer, T. R., Gerhardt, D. J., Dinger, M. E., Crawford, J., Trapnell, C., Jeddeloh, J. A., Mattick, J. S., Rinn, J. L., 2011. Targeted RNA sequencing reveals the deep complexity of the human transcriptome. Nat Biotechnol 30, 99-104, doi:10.1038/nbt.2024.

Miwa, K., Fujiwara, T., 2010. Boron transport in plants: co-ordinated regulation of transporters. Ann Bot 105, 1103-8, doi:10.1093/aob/mcq044.

Nawrocki, E. P., Kolbe, D. L., Eddy, S. R., 2009. Infernal 1.0: inference of RNA alignments. Bioinformatics 25, 1335-7, doi:10.1093/bioinformatics/btp157.

Nawrocki, E. P., Burge, S. W., Bateman, A., Daub, J., Eberhardt, R. Y., Eddy, S. R., Floden, E. W., Gardner, P. P., Jones, T. A., Tate, J., Finn, R. D., 2015. Rfam 12.0: updates to the RNA families database. Nucleic Acids Res 43, D130-7, doi:10.1093/nar/gku1063.

Ozhuner, E., Eldem, V., Ipek, A., Okay, S., Sakcali, S., Zhang, B., Boke, H., Unver, T., 2013. Boron stress responsive microRNAs and their targets in barley. PLoS One 8, e59543, doi:10.1371/journal.pone.0059543.

Paytuvi Gallart, A., Hermoso Pulido, A., Anzar Martinez de Lagran, I., Sanseverino, W., Aiese Cigliano, R., 2016. GREENC: a Wiki-based database of plant lncRNAs. Nucleic Acids Res 44, D1161-6, doi:10.1093/nar/gkv1215.

Qi, X., Xie, S., Liu, Y., Yi, F., Yu, J., 2013. Genome-wide annotation of genes and noncoding RNAs of foxtail millet in response to simulated drought stress by deep sequencing. Plant Mol Biol 83, 459-73, doi:10.1007/s11103-013-0104-6.

Quinlan, A. R., 2014. BEDTools: The Swiss-Army Tool for Genome Feature Analysis. Curr Protoc Bioinformatics 47, 1112 1-34, doi:10.1002/0471250953.bi1112s47.

Rinn, J. L., Chang, H. Y., 2012. Genome regulation by long noncoding RNAs. Annu Rev Biochem 81, 145-66, doi:10.1146/annurev-biochem-051410-092902.

Shafiq, S., Li, J., Sun, Q., 2016. Functions of plants long non-coding RNAs. Biochim Biophys Acta 1859, 155-62, doi:10.1016/j.bbagrm.2015.06.009.

Shuai, P., Liang, D., Tang, S., Zhang, Z., Ye, C. Y., Su, Y., Xia, X., Yin, W., 2014. Genome-wide identification and functional prediction of novel and drought- 
responsive lincRNAs in Populus trichocarpa. $\mathrm{J}$ Exp Bot 65, 4975-83, doi:10.1093/jxb/eru256.

Sousa, C., Johansson, C., Charon, C., Manyani, H., Sautter, C., Kondorosi, A., Crespi, M., 2001. Translational and structural requirements of the early nodulin gene enod40, a short-open reading frame-containing RNA, for elicitation of a cellspecific growth response in the alfalfa root cortex. Mol Cell Biol 21, 354-66, doi:10.1128/MCB.21.1.354-366.2001.

Szczesniak, M. W., Rosikiewicz, W., Makalowska, I., 2016. CANTATAdb: A Collection of Plant Long Non-Coding RNAs. Plant Cell Physiol 57, e8, doi:10.1093/pcp/pcv201.

Team, R. C., 2016. R: A language and environment for statistical computing. R Foundation for Statistical Computing, Vienna, Austria. 2015. URL h ttp. www. R-project. org.

The UniProt, C., 2017. UniProt: the universal protein knowledgebase. Nucleic Acids Res 45, D158-D169, doi:10.1093/nar/gkw1099.

Tombuloglu, G., Tombuloglu, H., Sakcali, M. S., Unver, T., 2015. High-throughput transcriptome analysis of barley (Hordeum vulgare) exposed to excessive boron. Gene 557, 71-81, doi:10.1016/j.gene.2014.12.012.

Tombuloglu, H., Kekec, G., Sakcali, M. S., Unver, T., 2013. Transcriptome-wide identification of R2R3-MYB transcription factors in barley with their boron responsive expression analysis. Mol Genet Genomics 288, 141-55, doi:10.1007/s00438-013-0740-1.

Tombuloglu, H., Ozcan, I., Tombuloglu, G., Sakcali, S., Unver, T., 2016. Aquaporins in Boron-Tolerant Barley: Identification, Characterization, and Expression Analysis. Plant Molecular Biology Reporter 34, 374-386.

Trapnell, C., Williams, B. A., Pertea, G., Mortazavi, A., Kwan, G., van Baren, M. J., Salzberg, S. L., Wold, B. J., Pachter, L., 2010. Transcript assembly and quantification by RNA-Seq reveals unannotated transcripts and isoform switching during cell differentiation. Nat Biotechnol 28, 511-5, doi:10.1038/nbt.1621.

Unver, T., Bozkurt, O., Akkaya, M. S., 2008. Identification of differentially expressed transcripts from leaves of the boron tolerant plant Gypsophila perfoliata L. Plant Cell Rep 27, 1411-22, doi:10.1007/s00299-008-0560-7.

Waterhouse, P. M., Hellens, R. P., 2015. Plant biology: Coding in non-coding RNAs. Nature 520, 41-2, doi:10.1038/nature14378.

Xie, C., Yuan, J., Li, H., Li, M., Zhao, G., Bu, D., Zhu, W., Wu, W., Chen, R., Zhao, Y., 2014. NONCODEv4: exploring the world of long non-coding RNA genes. Nucleic Acids Res 42, D98-103, doi:10.1093/nar/gkt1222.

Xin, M., Wang, Y., Yao, Y., Song, N., Hu, Z., Qin, D., Xie, C., Peng, H., Ni, Z., Sun, Q., 2011. Identification and characterization of wheat long non-protein coding RNAs responsive to powdery mildew infection and heat stress by using microarray analysis and SBS sequencing. BMC Plant Biol 11, 61, doi:10.1186/1471-2229-11-61.

Yanik, H., Turktas, M., Dundar, E., Hernandez, P., Dorado, G., Unver, T., 2013. Genome-wide identification of alternate bearing-associated microRNAs (miRNAs) in olive (Olea europaea L.). BMC Plant Biol 13, 10, doi:10.1186/1471-2229-13-10. 
Zhang, H., Chen, Z., Wang, X., Huang, Z., He, Z., Chen, Y., 2013. Long non-coding RNA: a new player in cancer. J Hematol Oncol 6, 37, doi:10.1186/1756-8722-637.

Zhou, H., Liu, Q., Li, J., Jiang, D., Zhou, L., Wu, P., Lu, S., Li, F., Zhu, L., Liu, Z., Chen, L., Liu, Y. G., Zhuang, C., 2012. Photoperiod- and thermo-sensitive genic male sterility in rice are caused by a point mutation in a novel noncoding RNA that produces a small RNA. Cell Res 22, 649-60, doi:10.1038/cr.2012.28.

\section{Figure legends}

\section{Figure 1. Chromosomal distribution of barley IncRNAs and their expression in}

normal and stress conditions. A. Total number of lncRNAs localized to each barley chromosome (from chromosome 1 to 7). B. Box plot representation of expression levels of lncRNAs before and after boron exposure in root and leaves. C. Expressional changes of lncRNAs across samples are being illustrated as heat map graph.

\section{Figure 2. Differentially expressed transcripts and GO term enrichment analysis.} A-B. Venn diagram of differentially expressed coding (A) and lncRNA (B) transcripts in leaf and root. C-D. Significant biological process (C) and molecular function (D) GO terms associated with differentially expressed coding transcripts.

Figure 3. Ion transport and response to stimulus associated IncRNAs. A-C. LncRNAs and coding transcripts related to ion transport (A) and response to stimulus (C) GO terms. LncRNAs and coding transcripts are represented as circles and round rectangles, respectively. Differentially expressed lncRNAs before and after boron exposure are shown as colored. Green colored lncRNAs are differentially expressed only in leaves. However, lcnRNAs are colored as red if they are differentially regulated 
1 in both root and leaves. LncRNAs and associated coding transcripts (co-expressed and

2 co-localized) are linked to each other with network edges. B-D. Heat map representation

3 of expression levels both lncRNAs and coding transcripts associated with ion transport

$4 \quad$ (B) and response to stimulus (D) GO terms.

5

6 Figure 4. Establishment of localization associated IncRNAs. A. Network

7 representation of establishment of localization related coding transcripts and lncRNAs

8 (the same color scheme as in Figure 3 is used). B. Expression levels of establishment of

9 localization GO term associated coding and lncRNAs.

11 Table:

12 Table 1. Computationally identified putative eTM sequences having potential to 13 act as miRNA sponge.

15 Supplementary Materials:

16 Table S1. Barley previously unannotated.transcrpits.bed

17 Table S2. Expression of lncRNAs and coding transcripts

18 Table S3. GO term enrichment analysis

19 Table S4. eTM sequences

20 Table S5. GO term enrichment analysis of eTM related miRNA target genes 


\section{Figure 1}

bioRxiv preprint doi: https://doi.org/10.1101/229559; this version posted December 5, 2017. The copyright holder for this preprint (which was not certified by peer review) is the author/funder, who has granted bioRxiv a license to display the preprint in perpetuity. It is made available A under aCC-BY-NC-ND 4.0 International license.


C

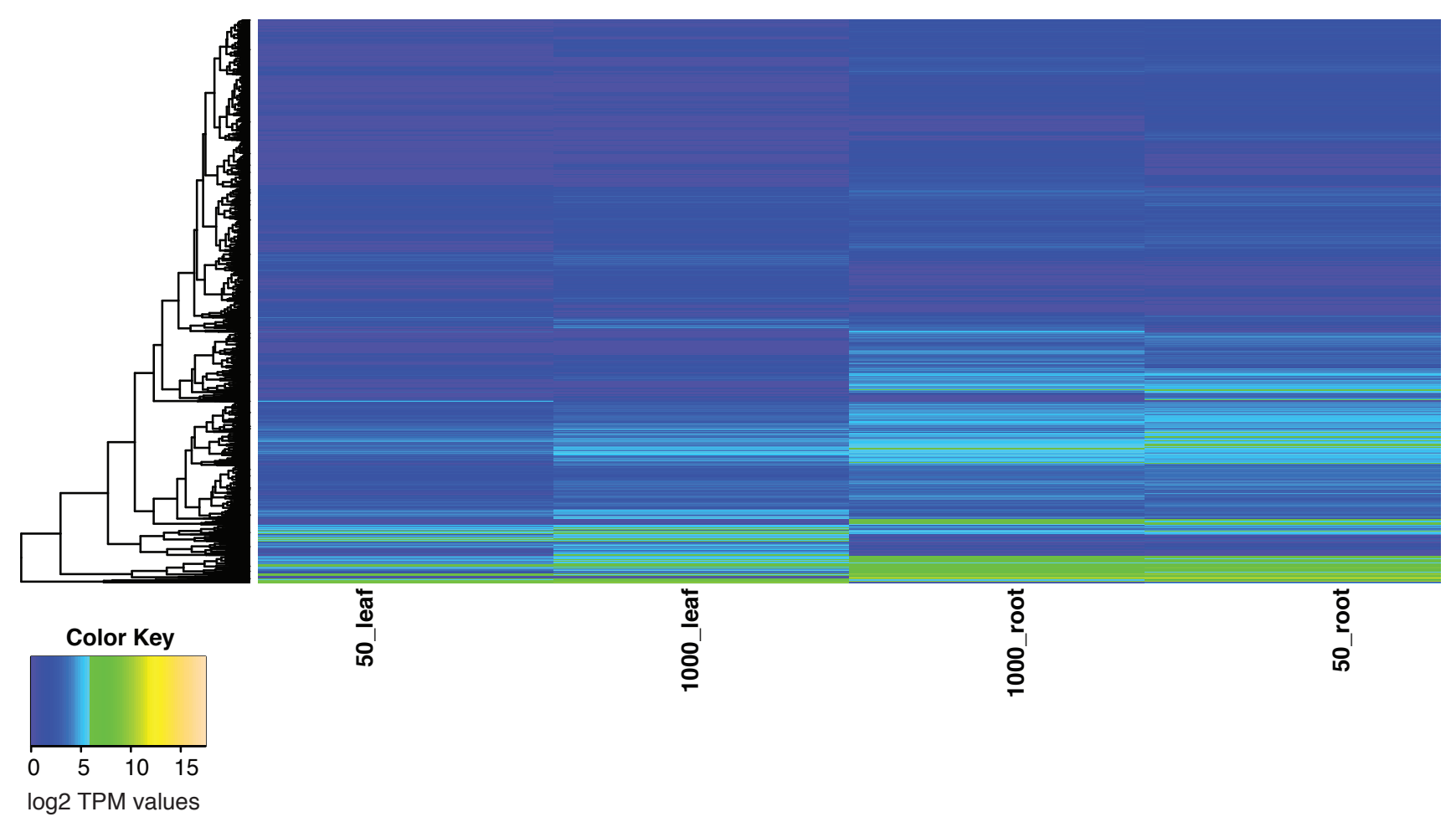




\section{Figure 2}

bipRxiv preprint doi: https://doi.org/10.1101/229559; this version posted Deeember 5, 2017. The copyright holder for this preprint (which was not certified by peet Root under aCC-BY-NC-ND 4.0 International liceisoe. Root

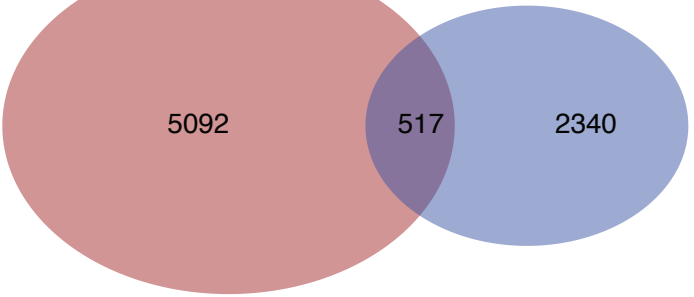

1799

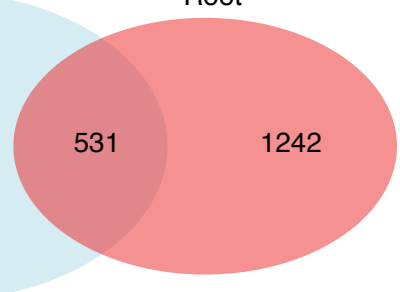

C
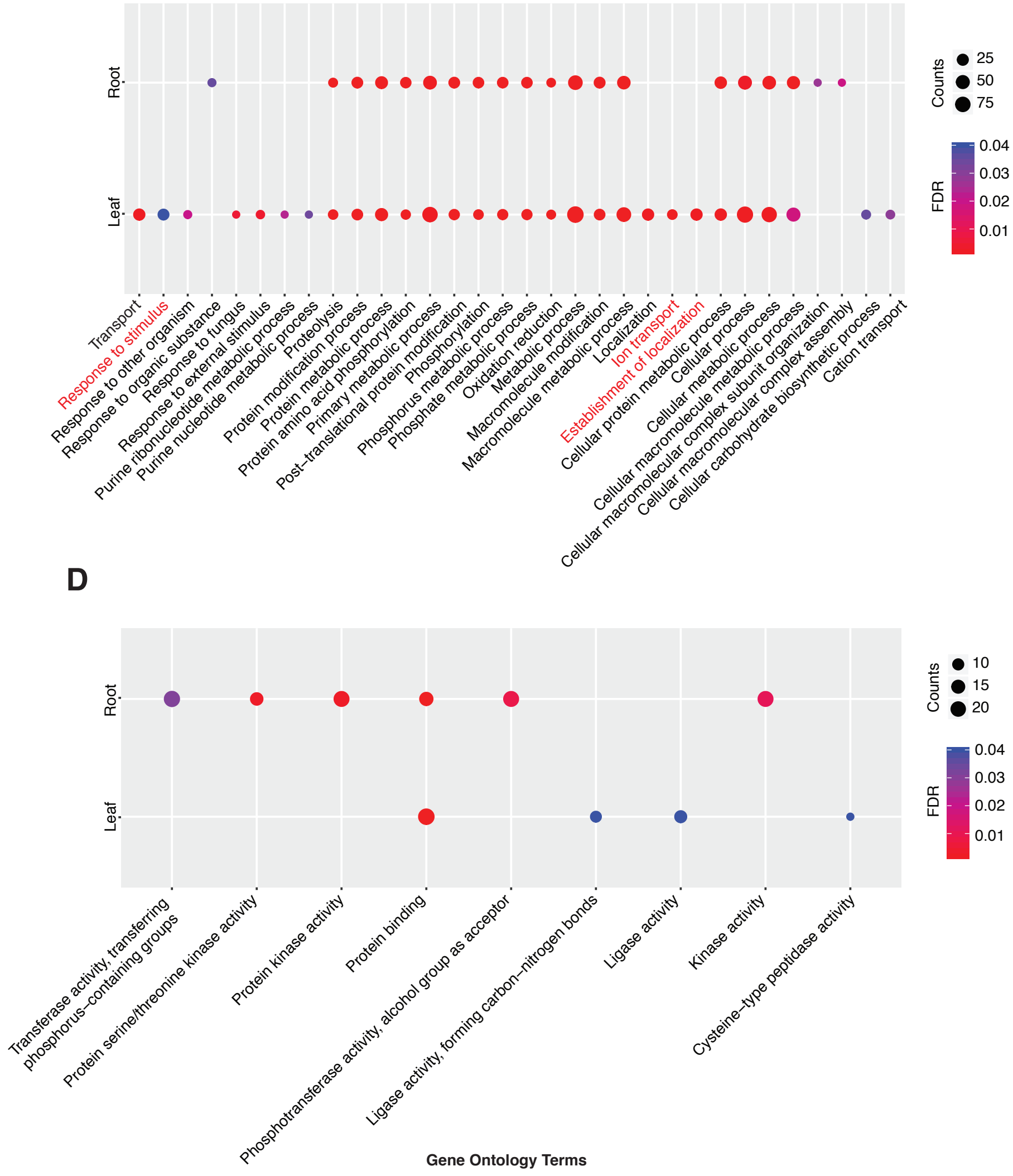
Figure 3

bioRxiv preprint doi: https://doi.org/10.1101/229559; this version posted December 5, 2017. The copyright holder for this preprint (which was

A not certified by peer review) is the author/funder, who has granted bioRxiv a license to display the preprint in perpetuity. It is made available

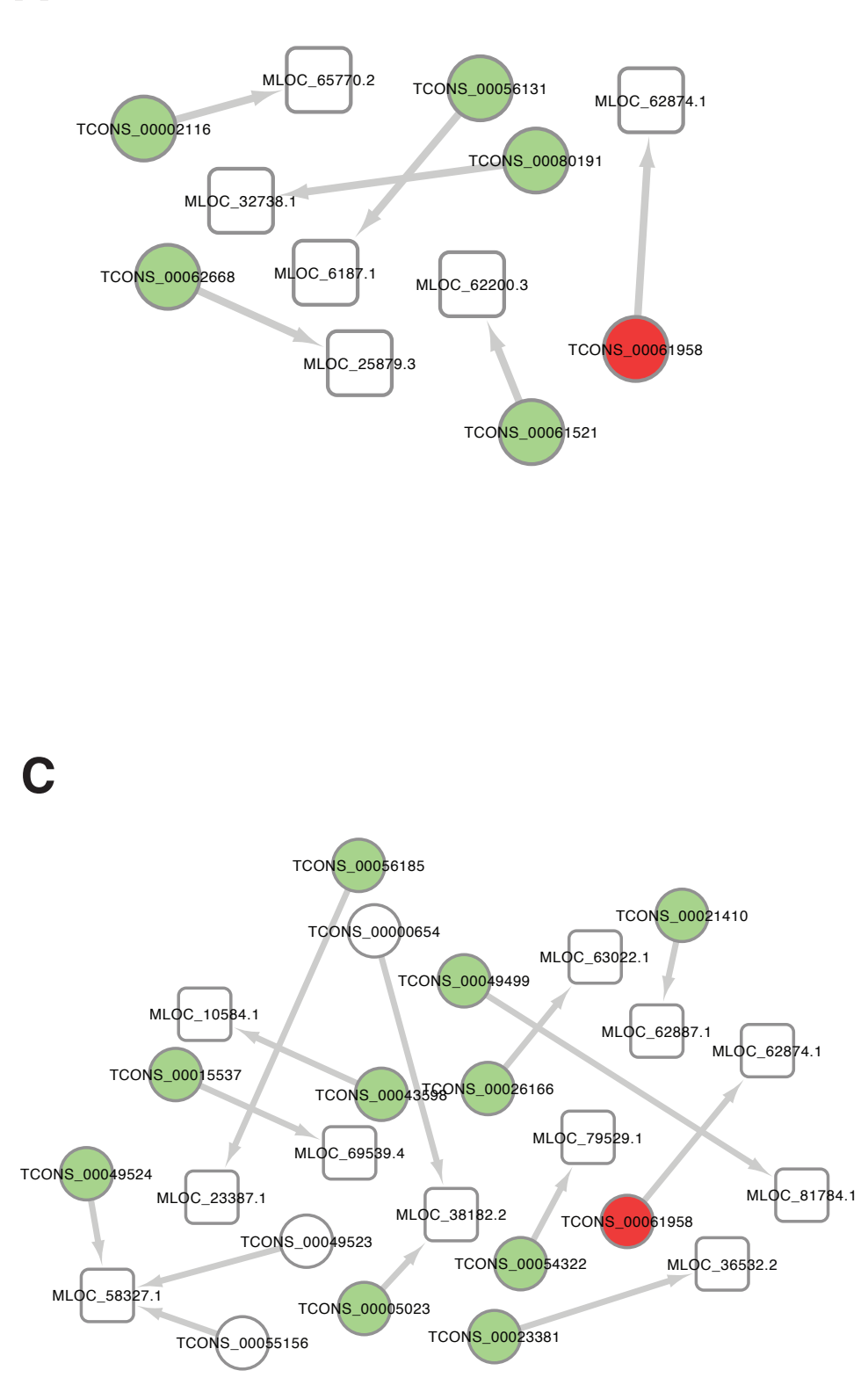
Bation

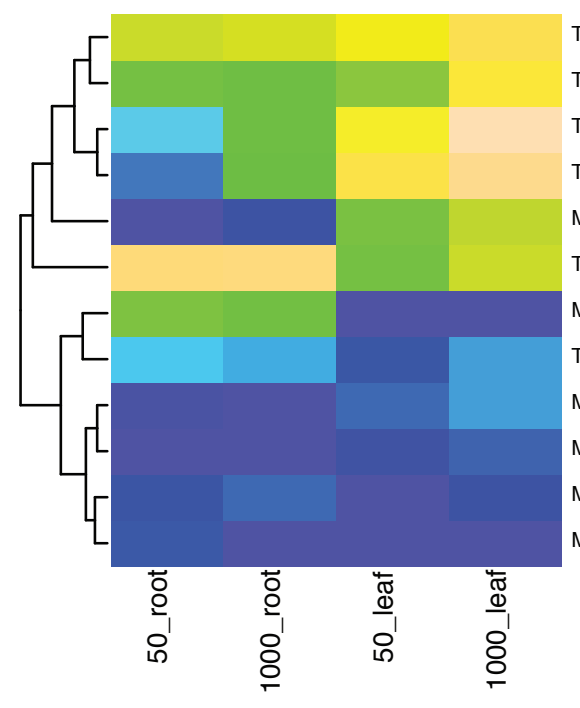

TCONS_00056131 TCONS_00002116 TCONS_00062668 TCONS_00061958 MLOC_62874.1 TCONS_00061521 MLOC_6187.1 TCONS_00080191 MLOC_65770.2 MLOC_25879.3 MLOC_62200.3 MLOC_32738.1

Color key

D
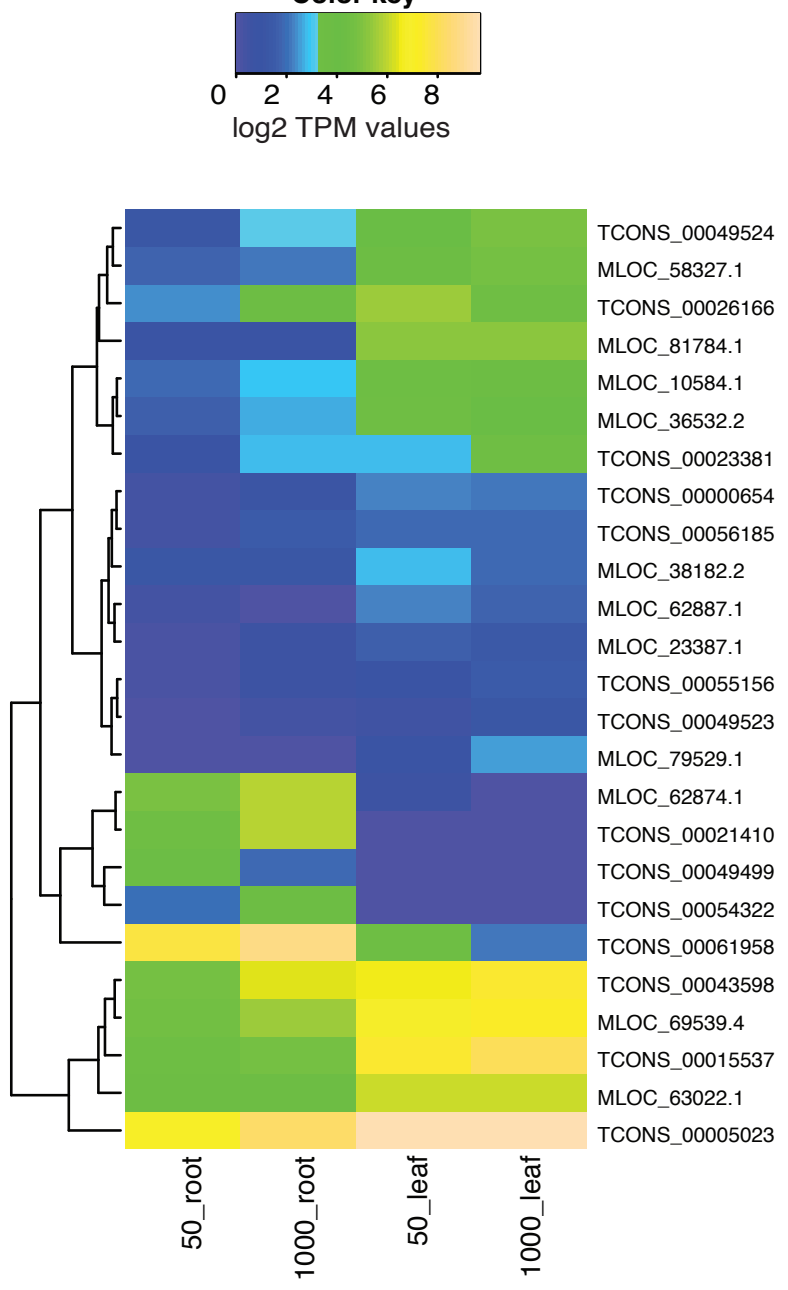


\section{Figure 4}

bioRxiv preprint doi: https://doi.org/10.1101/229559; this version posted December 5, 2017. The copyright holder for this preprint (which was A not certified by peer review) is the author/funder, who has granted bioRxiv a license to display the preprint in perpetuity. It is made available under aCC-BY-NC-ND 4.0 International license.

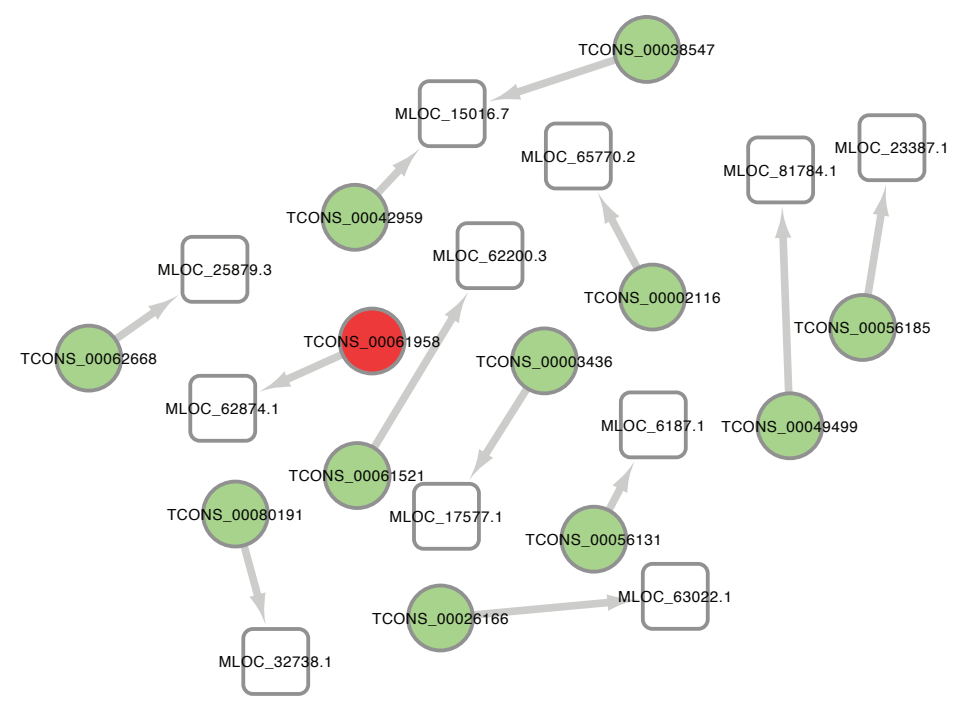

B



